\title{
REACTION-DIFFUSION WITH MEMORY IN THE MINIMAL STATE FRAMEWORK
}

\author{
MONICA CONTI, ELSA M. MARCHINI, AND VITTORINO PATA
}

Abstract. We consider the integrodifferential equation

$$
\partial_{t} u-\Delta u-\int_{0}^{\infty} \kappa(s) \Delta u(t-s) \mathrm{d} s+\varphi(u)=f
$$

arising in the Coleman-Gurtin theory of heat conduction with hereditary memory. Within a novel abstract framework, based on the notion of minimal state, we prove the existence of global and exponential attractors of optimal regularity and finite fractal dimension for the related semigroup of solutions.

\section{INTRODUCTION}

1.1. The equation. Let $\Omega \subset \mathbb{R}^{3}$ be a bounded domain with smooth boundary $\partial \Omega$. Introducing the strictly positive Dirichlet operator $A=-\Delta$ with domain

$$
\mathfrak{D}(A)=H^{2}(\Omega) \cap H_{0}^{1}(\Omega) \subset L^{2}(\Omega),
$$

we consider for $t>0$ the equation in the unknown variable $u: \Omega \times \mathbb{R} \rightarrow \mathbb{R}$ :

$$
\partial_{t} u+A\left[u+\int_{0}^{\infty} \kappa(s) u(t-s) \mathrm{d} s\right]+\varphi(u)=f .
$$

The following general assumptions on the constitutive terms are made:

A.1. The external force $f$ is time-independent and belongs to the space $L^{2}(\Omega)$.

A.2. The nonlinearity $\varphi \in \mathcal{C}^{2}(\mathbb{R})$ fulfills $\varphi(0)=0$, along with the growth restriction

$$
\left|\varphi^{\prime \prime}(u)\right| \leq c\left(1+|u|^{3}\right)
$$

and the dissipation condition

$$
\liminf _{|u| \rightarrow \infty} \varphi^{\prime}(u)>-\lambda_{1}
$$

where $\lambda_{1}>0$ is the first eigenvalue of $A$.

A.3. The convolution (or memory) kernel $\kappa$ is a nonnegative summable function of total mass $\int_{0}^{\infty} \kappa(s) \mathrm{d} s=1$ having the explicit form

$$
\kappa(s)=\int_{s}^{\infty} \mu(y) \mathrm{d} y
$$

Received by the editors October 17, 2011 and, in revised form, February 1, 2013. 2010 Mathematics Subject Classification. Primary 35B41, 35K05, 45K05, 47H20.

Key words and phrases. Heat conduction with memory, minimal state framework, solution semigroup, global attractor, exponential attractor. 
where $\mu \in L^{1}\left(\mathbb{R}^{+}\right)$is a nonincreasing (hence nonnegative) piecewise absolutely continuous function satisfying for some $M \geq 1$ and $\delta>0$ the inequality

$$
\mu(\tau+s) \leq M \mathrm{e}^{-\delta \tau} \mu(s), \quad \forall \tau, s>0 .
$$

The discontinuity points of $\mu$ (if any) form an increasing sequence $s_{n}$.

1.2. Physical motivations. Equation (1.1) rules the evolution of the temperature variation field $u$ in a homogeneous isotropic heat conductor $\Omega$ subject to hereditary memory, where the classical Fourier law for the heat flux $\boldsymbol{q}: \Omega \times \mathbb{R} \rightarrow \mathbb{R}^{3}$ is replaced by the Coleman-Gurtin constitutive law [5]

$$
\boldsymbol{q}(t)=-\alpha \nabla u(t)-\int_{0}^{\infty} \kappa(s) \nabla u(t-s) \mathrm{d} s
$$

based on the key assumption that the evolution of $\boldsymbol{q}$ is influenced by the past history of the temperature gradient (see also $13,17,18,20$ ). The constant $\alpha>0$ represents the instantaneous conductivity, whereas the convolution kernel $\kappa$ can be interpreted as a conductivity density, introducing delay effects in the model. The temperature is also required to satisfy the Dirichlet boundary condition

$$
u(\boldsymbol{x}, t)_{\mid \boldsymbol{x} \in \partial \Omega}=0,
$$

complying with the physical assumption that the boundary $\partial \Omega$ of the conductor is kept at null (equilibrium) temperature for all times. The thermal evolution is governed by the balance equation

$$
\partial_{t} e+\operatorname{div} \boldsymbol{q}=\mathfrak{F},
$$

where $e: \Omega \times \mathbb{R} \rightarrow \mathbb{R}$ is the internal energy of the system, while $\mathfrak{F}$ is a source term possibly depending on the temperature itself. For small variations of $u$ and its gradient, $e$ is well approximated by

$$
e(t)=e_{0}+c u(t)
$$

the function $e_{0}: \Omega \rightarrow \mathbb{R}$ being the internal energy at equilibrium and $c>0$ the specific heat. Hence, setting $\alpha=c=1$ for simplicity, (1.1) is recovered by choosing a nonlinear source of the form

$$
\mathfrak{F}(t)=-\varphi(u(t))+f .
$$

More generally, (1.1) serves as a model for the description of diffusive phenomena in the presence of delay mechanisms or memory effects.

Remark 1.1. It is also physically meaningful to consider, in place of the Dirichlet boundary condition, the no-flux boundary condition for $u$,

$$
\partial_{\nu} u(\boldsymbol{x}, t)_{\mid \boldsymbol{x} \in \partial \Omega}=0,
$$

where $\nu$ denotes the outer normal to $\Omega$. In this case, the linear version of (1.1) (with $\varphi \equiv 0)$ can be treated in a similar way, by considering spaces where the average of $u$ is conserved. In the nonlinear case, however, the dissipative character of the equation is lost, unless the operator $-\Delta$ (not coercive any longer) is replaced by $I-\Delta$ with Neumann boundary conditions. 
1.3. Asymptotic behavior. The longterm properties of equation (1.1) have been widely investigated in $[2,8,12$. In those papers, rephrasing the problem in the history framework of Dafermos [9], a dissipative solution semigroup is obtained, acting on a suitable phase space accounting for the past values of the variable $u$. Such a semigroup is shown to possess finite-dimensional global and exponential attractors of optimal regularity. Let alone the growth restriction (1.2) on the nonlinearity $\varphi$ (indeed, the polynomial order 5 is not attained in [8, 12]), the main improvement of the latest work 2] lies in condition (1.5) for the memory kernel $\mu$, in place of the less general

$$
\mu^{\prime}(s)+\delta \mu(s) \leq 0, \quad \forall s>0
$$

introduced in the seminal article [9] and commonly adopted in the literature thereafter.

Remark 1.2. Condition (1.5), first devised in [3], is easily seen to coincide with (1.6) if $M=1$. On the other hand, the picture becomes completely different when $M>1$. For instance, (1.6) prohibits $\mu$ to have (even local) flat zones, whereas any compactly supported $\mu$ fulfills (1.5) for some $M>1$. Furthermore, (1.5) turns out to be necessary for semigroups arising from systems with memory in order to exhibit uniform decay properties, such as the existence of absorbing sets (see [3]).

The aim of this work is a detailed analysis of equation (1.1) within a new theoretical scheme recently formalized in [16, the so-called minimal state framework. The need of a different perspective in connection with memory problems is motivated by an intrinsic weakness of the history approach and, more generally, of any other possible approach based on the knowledge of the past history of the variables in play at an arbitrarily given initial time. Indeed (cf. Section 4 below), such a request can be simultaneously unphysical and overabundant to determine the future evolution. On the contrary, the minimal state framework does not suffer from this drawback, providing a (minimal) description of the dynamics in terms of quantities which can be actually measured. In this spirit, paralleling [2], equation (1.1) is shown to generate a semigroup on a suitable Hilbert space, accounting this time for the "minimal state" (rather than the past history) of the variable $u$. Then, we prove the existence of finite-dimensional global and exponential attractors of optimal regularity, establishing an interesting connection between the two formulations.

1.4. Outline of the paper. The notation and the functional setting are introduced in the subsequent Section 2. In Section 3 we give the definition of a weak solution to (1.1). In Section 4 the equation is translated into an ODE in a Hilbert space $\mathcal{H}$ (the minimal state space), generating a strongly continuous semigroup $S(t)$ of solutions. Such solutions turn out to be weak solutions in the sense of the previous definition, as demonstrated in Section 5. Section 6 is devoted to the comparison between this novel approach and the well-known past history framework. The dissipativity features of the semigroup $S(t)$ are discussed in Section 7, showing the existence of bounded absorbing sets. The main result of the paper, concerned with the existence of global and exponential attractors for $S(t)$, is stated in Section 8 and proved in the final Section 9. 


\section{Functional Setting And notation}

We consider the scale of compactly nested Hilbert spaces

$$
\mathrm{H}^{q}=\mathfrak{D}\left(A^{\frac{q}{2}}\right), \quad q \in \mathbb{R},
$$

with inner products and norms given by

$$
\langle u, v\rangle_{q}=\left\langle A^{\frac{q}{2}} u, A^{\frac{q}{2}} v\right\rangle_{L^{2}(\Omega)} \quad \text { and } \quad\|u\|_{q}=\left\|A^{\frac{q}{2}} u\right\|_{L^{2}(\Omega)} .
$$

The index $q$ will be always omitted whenever zero. The symbol $\langle\cdot, \cdot\rangle$ will also stand for the duality product between $\mathrm{H}^{q}$ and its dual space $\mathrm{H}^{-q}$. We recall the well-known identities

$$
\mathrm{H}=L^{2}(\Omega), \quad \mathrm{H}^{1}=H_{0}^{1}(\Omega), \quad \mathrm{H}^{2}=H^{2}(\Omega) \cap H_{0}^{1}(\Omega),
$$

and the Poincaré inequality

$$
\lambda_{1}\|u\|^{2} \leq\|u\|_{1}^{2}, \quad \forall u \in \mathrm{H}^{1} .
$$

We will make use of two different classes of weighted $L^{2}$-spaces.

2.1. History spaces. For $q \in \mathbb{R}$ we introduce the history spaces ( $q$ is omitted if zero)

$$
\mathcal{M}^{q}=L_{\mu}^{2}\left(\mathbb{R}^{+} ; \mathrm{H}^{q+1}\right)
$$

endowed with the weighted $L^{2}$-inner products

$$
\left\langle\eta_{1}, \eta_{2}\right\rangle_{\mathcal{M}^{q}}=\int_{0}^{\infty} \mu(s)\left\langle\eta_{1}(s), \eta_{2}(s)\right\rangle_{q+1} \mathrm{~d} s,
$$

along with the extended history spaces

$$
\hat{\mathcal{H}}=\mathrm{H} \times \mathcal{M} \quad \text { and } \quad \hat{\mathcal{V}}=\mathrm{H}^{2} \times \mathcal{M}^{1} .
$$

For any $r \geq 0$ we define the ball

$$
\hat{\mathbb{B}}_{r}=\left\{\hat{z} \in \hat{\mathcal{H}}:\|\hat{z}\|_{\hat{\mathcal{H}}} \leq r\right\} .
$$

We will also consider the (linear) right-translation semigroup $R(t)$ on $\mathcal{M}$

$$
(R(t) \eta)(s)= \begin{cases}0, & s \leq t, \\ \eta(s-t), & s>t,\end{cases}
$$

whose infinitesimal generator is the linear operator

$$
T \eta=-\eta^{\prime} \quad \text { with domain } \quad \mathfrak{D}(T)=\left\{\eta \in \mathcal{M}: \eta^{\prime} \in \mathcal{M}, \eta(0)=0\right\},
$$

with the prime standing for a weak derivative.

2.2. Minimal state spaces. Calling $\varsigma \in(0, \infty]$ the supremum of the support of $\mu$, we define the new kernel $\nu:(0, \varsigma) \rightarrow \mathbb{R}^{+}$as

$$
\nu(\tau)=1 / \mu(\tau) \text {. }
$$

The assumptions on $\mu$ imply that $\nu$ is nondecreasing and piecewise absolutely continuous. Furthermore, rewriting (1.5) in terms of $\nu$ we get

$$
\nu(\tau-s) \leq M \mathrm{e}^{-\delta s} \nu(\tau), \quad \forall s<\tau<\varsigma .
$$

Remark 2.1. Aiming to describe the finite delay $(\varsigma<\infty)$ and the infinite delay $(\varsigma=\infty)$ cases in a unitary fashion, for any function $h=h(\tau)$ defined on $(0, \varsigma)$ we agree to put $h(\tau)=0$ whenever $\varsigma<\tau<\infty$. 
Then, for $q \in \mathbb{R}$, we introduce the (minimal) state spaces (again, $q$ is omitted if zero)

$$
\mathcal{S}^{q}=L_{\nu}^{2}\left(\mathbb{R}^{+} ; \mathrm{H}^{q+1}\right)
$$

with inner products

$$
\left\langle\xi_{1}, \xi_{2}\right\rangle_{\mathcal{S}^{q}}=\int_{0}^{\infty} \nu(\tau)\left\langle\xi_{1}(\tau), \xi_{2}(\tau)\right\rangle_{q+1} \mathrm{~d} \tau
$$

along with the extended state spaces

$$
\mathcal{H}=\mathrm{H} \times \mathcal{S} \quad \text { and } \quad \mathcal{V}=\mathrm{H}^{2} \times \mathcal{S}^{1} .
$$

As in the previous case, for any $r \geq 0$ we define the ball

$$
\mathbb{B}_{r}=\left\{z \in \mathcal{H}:\|z\|_{\mathcal{H}} \leq r\right\} .
$$

We will consider the (linear) left-translation semigroup $L(t)$ on $\mathcal{S}$

$$
(L(t) \xi)(\tau)=\xi(t+\tau),
$$

whose infinitesimal generator is the linear operator

$$
P \xi=\xi^{\prime} \quad \text { with domain } \quad \mathfrak{D}(P)=\left\{\xi \in \mathcal{S}: \xi^{\prime} \in \mathcal{S}\right\} .
$$

As shown in [7, 16], the operator $P$ fulfills

$$
\langle P \xi, \xi\rangle_{\mathcal{S}} \leq 0, \quad \forall \xi \in \mathfrak{D}(P),
$$

meaning that the semigroup is contractive. Actually, $L(t)$ is exponentially stable as well. Indeed, for every $\xi \in \mathcal{S}$ we infer from (2.1) that

$$
\|L(t) \xi\|_{\mathcal{S}}^{2}=\int_{t}^{\infty} \nu(\tau-t)\|\xi(\tau)\|_{1}^{2} \mathrm{~d} \tau \leq M \mathrm{e}^{-\delta t} \int_{t}^{\infty} \nu(\tau)\|\xi(\tau)\|_{1}^{2} \mathrm{~d} \tau \leq M \mathrm{e}^{-\delta t}\|\xi\|_{\mathcal{S}}^{2}
$$

2.3. The map $\Lambda$. The connection between the history and the state spaces has been devised in [7, 16. Supposing without loss of generality that $\mu$ is rightcontinuous, we denote by

$$
\mu_{n}=\mu\left(s_{n}^{-}\right)-\mu\left(s_{n}\right)>0
$$

the jump amplitudes at the (left) discontinuity points $s_{n}$. Setting for any $\eta \in \mathcal{M}$

$$
(\Lambda \eta)(\tau)=-\int_{0}^{\infty} \mu^{\prime}(\tau+s) \eta(s) \mathrm{d} s+\sum_{\tau<s_{n}} \mu_{n} \eta\left(s_{n}-\tau\right),
$$

the following result is proved.

Theorem 2.2. The map $\boldsymbol{\Lambda}$ defined by

$$
(u, \eta) \mapsto \boldsymbol{\Lambda}(u, \eta)=(u, \Lambda \eta)
$$

is a bounded linear operator of unitary norm from $\hat{\mathcal{H}}$ into $\mathcal{H}$, as well as from $\hat{\mathcal{V}}$ into $\mathcal{V}$. Moreover, we have the equality

$$
\int_{0}^{\infty} \mu(s) \eta(s) \mathrm{d} s=\int_{0}^{\infty}(\Lambda \eta)(\tau) \mathrm{d} \tau, \quad \forall \eta \in \mathcal{M} .
$$

A further technical lemma will be needed (see [7, Lemma 2.1]). 
Lemma 2.3. Let $p: \mathbb{R}^{+} \rightarrow \mathrm{H}^{1}$ satisfy

$$
\int_{0}^{\infty} \mu(\tau+s)\|p(s)\|_{1} \mathrm{~d} s<\infty, \quad \forall \tau>0 .
$$

Assume also that its primitive $\tilde{p}(s)=\int_{0}^{s} p(y) \mathrm{d} y$ belongs to $\mathcal{M}$. Then

$$
(\Lambda \tilde{p})(\tau)=\int_{0}^{\infty} \mu(\tau+s) p(s) \mathrm{d} s .
$$

Remark 2.4. If $p \in L^{1}\left(\mathbb{R}^{+} ; \mathrm{H}^{1}\right)$ the hypotheses of the lemma are easily verified.

\section{WEAK SOLUTIONS}

The evolution of (1.1) is influenced by the past history of the variable $u$. Accordingly, at the initial time $t=0$, the exact value of the convolution integral

$$
\int_{0}^{\infty} \kappa(s) u(-s) \mathrm{d} s
$$

is needed. To this end, after the seminal work of Dafermos 9, a widely used strategy is to regard the past history of $u$ as an initial datum of the problem, i.e.

$$
u(0)=u_{0} \quad \text { and } \quad u(-s)_{\mid s>0}=g_{0}(s),
$$

where $u_{0}: \Omega \rightarrow \mathbb{R}$ and $g_{0}: \Omega \times \mathbb{R}^{+} \rightarrow \mathbb{R}$ are assumed to be known. In that case, introducing the function

$$
G_{0}(t)=\int_{0}^{\infty} \kappa(t+s) g_{0}(s) \mathrm{d} s, \quad t \geq 0,
$$

equation (1.1) can be reformulated as

$$
\partial_{t} u+A\left[u+\int_{0}^{t} \kappa(s) u(t-s) \mathrm{d} s+G_{0}\right]+\varphi(u)=f .
$$

This leads to the following quite natural notion of a (weak) solution.

Definition 3.1. Let $u_{0} \in \mathrm{H}$ and let $g_{0}$ be such that the corresponding $G_{0}$ in (3.2) belongs to $\mathrm{H}^{1}$ for almost every $t$. A function

$$
u \in \mathcal{C}([0, \infty), \mathrm{H}) \cap L_{\mathrm{loc}}^{2}\left(0, \infty ; \mathrm{H}^{1}\right)
$$

is a solution to (1.1) with initial condition (3.1) if $u(0)=u_{0}$ and

$\left\langle\partial_{t} u(t), v\right\rangle+\langle u(t), v\rangle_{1}+\int_{0}^{t} \kappa(s)\langle u(t-s), v\rangle_{1} \mathrm{~d} s+\left\langle G_{0}(t), v\right\rangle_{1}+\langle\varphi(u(t)), v\rangle=\langle f, v\rangle$,

for every test $v \in \mathrm{H}^{1}$ and almost every $t>0$.

A quite successful way to overcome the difficulties arising from the nonlocal character of the equation is setting the problem in the so-called history framework of Dafermos [9]. More precisely, by introducing for $t \geq 0$ and $s>0$ the integrated past history $\eta=\eta^{t}(s)$ of the variable $u$, formally defined as

$$
\eta^{t}(s)=\int_{0}^{s} u(t-y) \mathrm{d} y,
$$


the original equation (1.1) translates into the system in the unknown variables $u=u(t)$ and $\eta=\eta^{t}(s)$,

$$
\left\{\begin{array}{l}
\partial_{t} u+A\left[u+\int_{0}^{\infty} \mu(s) \eta(s) \mathrm{d} s\right]+\varphi(u)=f, \\
\partial_{t} \eta(s)=-\partial_{s} \eta(s)+u .
\end{array}\right.
$$

In turn, the initial conditions (3.1) become

$$
u(0)=u_{0} \quad \text { and } \quad \eta^{0}(s)=\int_{0}^{s} g_{0}(y) \mathrm{d} y .
$$

By adopting this strategy, several results have been proved in the last years, especially with regard to the asymptotic behavior of solutions (see e.g. [2, 8, 13, 17, 18, 20 ).

\section{The Minimal STATE FRAMEWORK}

4.1. The initial condition problem. A closer look at (3.3) tells us that, besides $u_{0}$, all that is needed to determine the evolution of $u$ is the knowledge of the function $G_{0}$ in (3.2). This points out a structural theoretical drawback of the history approach: two different past histories $g_{0}$ may lead to the same $G_{0}(t)$, hence to the same solution $u(t)$ for $t \geq 0$ (see [10,11). In other words, the "initial datum" $g_{0}$ may not be recoverable by the evolution of the system, and so is not a physical (measurable) quantity.

Remark 4.1. An immediate example is given by the memory kernel $\kappa(s)=\mathrm{e}^{-s}$, for which (3.2) reads

$$
G_{0}(t)=\mathrm{e}^{-t} \int_{0}^{\infty} \mathrm{e}^{-s} g_{0}(s) \mathrm{d} s .
$$

It is apparent here that infinitely many $g_{0}$ produce the same function $G_{0}$.

Such an obstacle can be overcome by adopting a different theoretical scheme, recently devised in [16], based on the notion of minimal state: an additional variable accounting for the past history which contains the necessary and sufficient information determining the future dynamics.

4.2. Heuristic derivation of the scheme. For $t \geq 0$ and $\tau>0$ we introduce an auxiliary variable $\xi=\xi^{t}(\tau)$, which we call a minimal state, formally defined as

$$
\xi^{t}(\tau)=\int_{0}^{\infty} \mu(\tau+s) u(t-s) \mathrm{d} s,
$$

which (again, formally) satisfies the relations

$$
\partial_{t} \xi^{t}(\tau)=\partial_{\tau} \xi^{t}(\tau)+\mu(\tau) u(t)
$$

and

$$
\int_{0}^{\infty} \xi^{t}(\tau) \mathrm{d} \tau=\int_{0}^{\infty} \kappa(s) u(t-s) \mathrm{d} s
$$

Hence, we rewrite (1.1) into an evolution system in the unknown variables $u=u(t)$ and $\xi=\xi^{t}(\tau)$

$$
\left\{\begin{array}{l}
\partial_{t} u+A\left[u+\int_{0}^{\infty} \xi(\tau) \mathrm{d} \tau\right]+\varphi(u)=f \\
\partial_{t} \xi(\tau)=\partial_{\tau} \xi(\tau)+\mu(\tau) u
\end{array}\right.
$$


where the initial condition (3.1) translates into

$$
u(0)=u_{0} \quad \text { and } \quad \xi^{0}(\tau)=\int_{0}^{\infty} \mu(\tau+s) g_{0}(s) \mathrm{d} s .
$$

Such a description meets the sought after minimality requirement. Indeed, once the initial state $\xi^{0}$ is assigned, we can express (4.1) in the equivalent form

$$
\xi^{t}(\tau)=\xi^{0}(t+\tau)+\int_{0}^{t} \mu(\tau+s) u(t-s) \mathrm{d} s .
$$

Then, plugging (4.3) into the first equation of (4.2), we deduce for every $t \geq 0$ the equality

$$
\int_{t}^{\infty} \xi^{0}(\tau) \mathrm{d} \tau=U(t)
$$

for some function $U$ depending only on $u(t)_{\mid t \geq 0}$. Accordingly, the knowledge of $u(t)$ for all $t \geq 0$ uniquely determines $\xi^{0}$, and so $\xi^{t}$ by again invoking (4.3).

4.3. The semigroup. In order to give the heuristic scheme a rigorous interpretation in a suitable functional setting, the idea to view (4.2) as the ODE in $\mathcal{H}$ :

$$
\left\{\begin{array}{l}
\dot{u}+A\left[u+\int_{0}^{\infty} \xi(\tau) \mathrm{d} \tau\right]+\varphi(u)=f \\
\dot{\xi}(\tau)=(P \xi)(\tau)+\mu(\tau) u .
\end{array}\right.
$$

A well-posedness result holds.

Theorem 4.2. Equation (4.4) generates a solution semigroup $S(t): \mathcal{H} \rightarrow \mathcal{H}$. Thus, for every $t \geq 0$ and every $z=\left(u_{0}, \xi_{0}\right) \in \mathcal{H}$,

$$
S(t) z=\left(u(t), \xi^{t}\right)
$$

is the unique solution at time $t$ to (4.4) with initial datum $z$. In addition,

$$
u \in L_{\text {loc }}^{2}\left(0, \infty ; \mathrm{H}^{1}\right),
$$

while $\xi$ fulfills the explicit representation formula (4.3) with $\xi^{0}=\xi_{0}$.

We omit the proof of the theorem, which is essentially identical to the one of the existence and uniqueness result in [8] (see also [2]), up to recasting the arguments in the minimal state framework. We only observe that, once $u$ is given, the explicit formula (4.3) is obtained from the second equation of (4.4) by applying the standard variation of constants method.

Proposition 4.3. The continuous dependence estimate

$$
\left\|S(t) z_{1}-S(t) z_{2}\right\|_{\mathcal{H}} \leq \mathrm{e}^{\ell t}\left\|z_{1}-z_{2}\right\|_{\mathcal{H}}
$$

holds for some $\ell \geq 0$ and every $z_{1}, z_{2} \in \mathcal{H}$.

Proof. The difference

$$
Z(t)=\left(w(t), \zeta^{t}\right)=S(t) z_{1}-S(t) z_{2}
$$

fulfills the problem

$$
\left\{\begin{array}{l}
\dot{w}+A\left[w+\int_{0}^{\infty} \zeta(\tau) \mathrm{d} \tau\right]+\varphi\left(u_{1}\right)-\varphi\left(u_{2}\right)=0 \\
\dot{\zeta}(\tau)=(P \zeta)(\tau)+\mu(\tau) w \\
Z(0)=z_{1}-z_{2}
\end{array}\right.
$$


where $u_{\imath}(t)$ is the first component of $S(t) z_{\imath}$. Working as usual in a regularization scheme, we multiply the first equation by $w$ in $\mathrm{H}$ and the second one by $\zeta$ in $\mathcal{S}$, thus obtaining

$$
\frac{\mathrm{d}}{\mathrm{d} t}\|Z\|_{\mathcal{H}}^{2}+2\left\langle\varphi\left(u_{1}\right)-\varphi\left(u_{2}\right), w\right\rangle=-2\|w\|_{1}^{2}+2\langle P \zeta, \zeta\rangle_{\mathcal{S}} \leq 0
$$

having used (2.2) in the latter inequality. Besides, (1.3) implies that $\varphi^{\prime} \geq-\ell$ for some $\ell \geq 0$. Consequently,

$$
2\left\langle\varphi\left(u_{1}\right)-\varphi\left(u_{2}\right), w\right\rangle \geq-2 \ell\|w\|^{2} \geq-2 \ell\|Z\|_{\mathcal{H}}^{2} .
$$

Summarizing, we end up with

$$
\frac{\mathrm{d}}{\mathrm{d} t}\|Z\|_{\mathcal{H}}^{2} \leq 2 \ell\|Z\|_{\mathcal{H}}^{2}
$$

and the sought after inequality follows from the Gronwall lemma.

\section{RECOVERING the ORIGINAL EQUATION}

We now clarify the correspondence between the original problem (1.1) and its reformulation in the minimal state framework. We begin with a definition.

Definition 5.1. A measurable function $G: \mathbb{R}^{+} \rightarrow \mathrm{H}^{1}$ is called a state function if

$$
G(t)=\int_{0}^{\infty} \kappa(t+s) g(s) \mathrm{d} s
$$

for some measurable $g: \mathbb{R}^{+} \rightarrow \mathrm{H}^{1}$. We denote by $\mathbb{S}$ the space of state functions.

Remark 5.2. Note that $G(t)$ is defined in $\mathrm{H}^{1}$ (as a Bochner integral) for every $t>0$. Indeed, given any $t>0$, there is a time $t_{0} \leq t$ such that $G\left(t_{0}\right) \in \mathrm{H}^{1}$. Since $\kappa$ is nonincreasing, this is the same as saying that

$$
\int_{0}^{\infty} \kappa(t+s)\|g(s)\|_{1} \mathrm{~d} s \leq \int_{0}^{\infty} \kappa\left(t_{0}+s\right)\|g(s)\|_{1} \mathrm{~d} s<\infty .
$$

The space $\mathbb{S}$ fulfills some regularity properties.

Lemma 5.3. Let $G \in \mathbb{S}$. For any $g$ complying with (5.1) the identities

$$
G(t)=\int_{t}^{\infty}\left[\int_{0}^{\infty} \mu(\tau+s) g(s) \mathrm{d} s\right] \mathrm{d} \tau=\int_{0}^{\infty} \mu(t+s)\left[\int_{0}^{s} g(y) \mathrm{d} y\right] \mathrm{d} s
$$

occur for every $t>0$.

Proof. Select $g$ for which the representation (5.1) holds. By virtue of (1.4) we have the equality

$$
\kappa(t+s)=\int_{t}^{\infty} \mu(\tau+s) \mathrm{d} \tau,
$$

and exchanging the order of integration we obtain

$$
G(t)=\int_{t}^{\infty}\left[\int_{0}^{\infty} \mu(\tau+s) g(s) \mathrm{d} s\right] \mathrm{d} \tau .
$$

Writing instead

$$
\kappa(t+s)=\int_{s}^{\infty} \mu(t+\tau) \mathrm{d} \tau
$$


we are led to

$$
G(t)=\int_{0}^{\infty}\left[\int_{s}^{\infty} \mu(t+\tau) g(s) \mathrm{d} \tau\right] \mathrm{d} s=\int_{0}^{\infty} \mu(t+s)\left[\int_{0}^{s} g(y) \mathrm{d} y\right] \mathrm{d} s .
$$

This finishes the proof.

Differentiating the first identity of the lemma we get an immediate corollary.

Corollary 5.4. If $G \in \mathbb{S}$, then $G^{\prime} \in L^{1}\left(t, \infty ; \mathrm{H}^{1}\right)$ for all $t>0$ and

$$
G(t)=-\int_{t}^{\infty} G^{\prime}(\tau) \mathrm{d} \tau .
$$

Moreover, for any $g$ satisfying (5.1), the derivative $G^{\prime}$ has the explicit form

$$
G^{\prime}(\tau)=-\int_{0}^{\infty} \mu(\tau+s) g(s) \mathrm{d} s .
$$

In particular, the space inclusion $\mathbb{S} \subset \mathcal{C}_{0}\left([t, \infty), \mathrm{H}^{1}\right)$ holds for every $t>0$.

At this point, we can rephrase Definition 3.1 in a more convenient way by considering initial conditions of the form

$$
\left(u_{0}, G_{0}\right) \in \mathrm{H} \times \mathbb{S},
$$

complying with the fact that $G_{0}$, rather than $g_{0}$, is the correct initial datum describing the past history of $u$, for it contains all the information determining the future dynamics. In fact, we will restrict ourselves to a particular (albeit quite general) class of state functions.

Definition 5.5. A function $G \in \mathbb{S}$ is a proper state function whenever its derivative $G^{\prime}$ is an element of $\mathcal{S}$. We call $\mathbb{S}_{\mathrm{p}}$ the space of proper state functions.

We are now in a position to establish the link between the two different formulations of the problem.

Proposition 5.6. Assume that $\left(u_{0}, G_{0}\right) \in \mathrm{H} \times \mathbb{S}_{\mathrm{p}}$. Then $u$ is a solution to (1.1) with initial conditions $\left(u_{0}, G_{0}\right)$ if and only if

$$
\left(u(t), \xi^{t}\right)=S(t)\left(u_{0}, \xi_{0}\right),
$$

where $\xi_{0}=-G_{0}^{\prime}$ and $\xi^{t}$ as in (4.3).

Proof. Having $G_{0}^{\prime}=-\xi_{0}$, Corollary 5.4 entails

$$
G_{0}(t)=\int_{t}^{\infty} \xi_{0}(\tau) \mathrm{d} \tau=\int_{0}^{\infty} \xi_{0}(t+\tau) \mathrm{d} \tau
$$

Since in both cases $u \in L^{1}\left(0, t ; \mathrm{H}^{1}\right)$ for every $t>0$, we learn from (1.4) that

$$
\int_{0}^{t} \kappa(s) u(t-s) \mathrm{d} s=\int_{0}^{\infty}\left(\int_{0}^{t} \mu(\tau+s) u(t-s) \mathrm{d} s\right) \mathrm{d} \tau .
$$

In summary, we draw the identity

$$
\int_{0}^{t} \kappa(s) u(t-s) \mathrm{d} s+G_{0}(t)=\int_{0}^{\infty} \xi^{t}(\tau) \mathrm{d} \tau,
$$

with $\xi^{t}$ given by (4.3). The claim follows by comparing (3.3) and (4.4).

We end with a sufficient condition for a state function to be proper. 
Proposition 5.7. A state function $G$ lies in $\mathbb{S}_{\mathrm{p}}$ whenever there exists a representation (5.1) for some $g$ whose primitive $\tilde{g}(s)=\int_{0}^{s} g(y) \mathrm{d} y$ belongs to the space $\mathcal{M}$.

Proof. By Corollary 5.4 we know that for every $\tau>0$,

$$
G^{\prime}(\tau)=-\int_{0}^{\infty} \mu(\tau+s) g(s) \mathrm{d} s \in \mathrm{H}^{1} .
$$

Therefore, as $\tilde{g} \in \mathcal{M}$, Lemma 2.3 yields the equality

$$
G^{\prime}(\tau)=-(\Lambda \tilde{g})(\tau)
$$

implying in turn $G^{\prime} \in \mathcal{S}$.

\section{Minimal State versus history}

Next, we dwell on the comparison between the minimal state and the past history formulations of problem (1.1). Indeed, paralleling the discussion leading to (4.4), the formal evolution system (3.4) is interpreted as the ODE in the extended history space $\hat{\mathcal{H}}$ :

$$
\left\{\begin{array}{l}
\dot{u}+A\left[u+\int_{0}^{\infty} \mu(s) \eta(s) \mathrm{d} s\right]+\varphi(u)=f \\
\dot{\eta}(s)=(T \eta)(s)+u .
\end{array}\right.
$$

As shown in [8, equation (6.1) generates a solution semigroup $\hat{S}(t): \hat{\mathcal{H}} \rightarrow \hat{\mathcal{H}}$. Besides, for every solution

$$
\hat{S}(t) \hat{z}=\left(u(t), \eta^{t}\right) \quad \text { with } \quad \hat{z}=\left(u_{0}, \eta_{0}\right) \in \hat{\mathcal{H}},
$$

we have that $u \in L_{\text {loc }}^{2}\left(0, \infty ; \mathrm{H}^{1}\right)$, while $\eta$ fulfills the representation formula

$$
\eta^{t}(s)= \begin{cases}\int_{0}^{s} u(t-y) \mathrm{d} y, & s \leq t, \\ \eta_{0}(s-t)+\int_{0}^{t} u(t-y) \mathrm{d} y, & s>t .\end{cases}
$$

The link between the history and the minimal state formulations is detailed in the next proposition, showing in particular that the state approach describes the dynamics in greater generality.

Proposition 6.1. For every $\hat{z} \in \hat{\mathcal{H}}$ the following equality holds:

$$
S(t) \boldsymbol{\Lambda} \hat{z}=\boldsymbol{\Lambda} \hat{S}(t) \hat{z} .
$$

Proof. Given a solution

$$
\hat{S}(t) \hat{z}=\left(u(t), \eta^{t}\right) \quad \text { with } \quad \hat{z}=\left(u_{0}, \eta_{0}\right) \in \hat{\mathcal{H}},
$$

let us define

$$
\xi^{t}(\tau)=\left(\Lambda \eta^{t}\right)(\tau) \quad \text { and } \quad \xi_{0}=\Lambda \eta_{0} .
$$

By Theorem 2.2 we have the equality

$$
\int_{0}^{\infty} \mu(s) \eta^{t}(s) \mathrm{d} s=\int_{0}^{\infty} \xi^{t}(\tau) \mathrm{d} \tau .
$$


Accordingly, the pair $\left(u(t), \xi^{t}\right)$ fulfills the first equation of (4.4). In order to verify the second equation, it is convenient to extend $u$ equal to zero on $\mathbb{R}^{-}$. Then we can give (6.2) the equivalent form

$$
\eta^{t}(s)=\left(R(t) \eta_{0}\right)(s)+\int_{0}^{s} u(t-y) \mathrm{d} y
$$

By the very definition of $\Lambda$, we easily get

$$
\left(\Lambda R(t) \eta_{0}\right)(\tau)=\left(L(t) \xi_{0}\right)(\tau)=\xi_{0}(t+\tau),
$$

while an application of Lemma 2.3 with

$$
p=u(t-\cdot) \in L^{1}\left(\mathbb{R}^{+} ; \mathrm{H}^{1}\right)
$$

yields the identity

$$
(\Lambda \tilde{p})(\tau)=\int_{0}^{\infty} \mu(\tau+s) p(s) \mathrm{d} s=\int_{0}^{t} \mu(\tau+s) u(t-s) \mathrm{d} s .
$$

Summarizing, the function $\xi^{t}=\Lambda \eta^{t}$ fulfills the representation formula (4.3), and so the second equation of (4.4).

\section{Dissipative estimates}

We now turn to the main focus of this work: the analysis of the global asymptotic properties of the solutions to (4.4). As is customary, the first step is proving the dissipative character of the semigroup $S(t)$, ensured by the existence of a bounded set $\mathfrak{B} \subset \mathcal{H}$, called absorbing set, is able to capture all trajectories originating from any given bounded set of initial data in finite time.

Theorem 7.1. There exist $\varepsilon>0$ and $c \geq 0$ such that, for every $z \in \mathbb{B}_{r}$ and every $t \geq 0$,

$$
\|S(t) z\|_{\mathcal{H}}^{2} \leq 2\|z\|_{\mathcal{H}}^{2} \mathrm{e}^{-\varepsilon t}+c .
$$

Remark 7.2. For example, the ball

$$
\mathfrak{B}=\mathbb{B}_{\sqrt{2 c}}
$$

is an absorbing set for $S(t)$. Indeed, for every $r \geq 0$, Theorem 7.1 tells that

$$
S(t) \mathbb{B}_{r} \subset \mathfrak{B}, \quad \forall t \geq t_{r},
$$

where $t_{r}=\frac{1}{\varepsilon}\left(\log \frac{2 r^{2}}{c}\right)^{+} \geq 0$ is the entering time of $\mathbb{B}_{r}$ into $\mathfrak{B}$.

Proof of Theorem 7.1. Calling

$$
E(t)=\|S(t) z\|_{\mathcal{H}}^{2}
$$

we multiply within a regularization scheme (4.4) by $(u, \xi)$ in $\mathcal{H}$ and we get

$$
\frac{\mathrm{d}}{\mathrm{d} t} E+2\|u\|_{1}^{2}+2\langle\varphi(u), u\rangle-2\langle f, u\rangle=2\langle P \xi, \xi\rangle_{\mathcal{S}} .
$$

In light of (1.3), completely standard calculations together with the Poincaré inequality entail the control

$$
2\langle\varphi(u), u\rangle-2\langle f, u\rangle \geq-(2-\alpha)\|u\|_{1}^{2}-b
$$

for some $\alpha>0$ (possibly very small) and some $b \geq 0$. Thus, recalling (2.2), we end up with

$$
\frac{\mathrm{d}}{\mathrm{d} t} E+\alpha\|u\|_{1}^{2} \leq b
$$


Defining the integrated kernel

$$
\varrho(\tau)=\int_{0}^{\tau} \nu(s) \mathrm{d} s
$$

which satisfies (due to (2.1))

$$
\int_{0}^{\tau} \nu(s) \mathrm{d} s \leq M \nu(\tau) \int_{0}^{\tau} \mathrm{e}^{-\delta(\tau-s)} \mathrm{d} s \leq \frac{M}{\delta} \nu(\tau),
$$

we introduce the functional

$$
\Psi(t)=2 \int_{0}^{\infty} \varrho(\tau)\left\|\xi^{t}(\tau)\right\|_{1}^{2} \mathrm{~d} \tau
$$

By (7.2), we find the uniform-in-time control

$$
\Psi(t) \leq \frac{2 M}{\delta}\left\|\xi^{t}\right\|_{\mathcal{S}}^{2} \leq \frac{2 M}{\delta} E(t)
$$

Taking the time derivative of $\Psi$ and using the second equation of (4.4) we obtain

$$
\frac{\mathrm{d}}{\mathrm{d} t} \Psi=2 \int_{0}^{\infty} \varrho(\tau) \frac{\mathrm{d}}{\mathrm{d} \tau}\|\xi(\tau)\|_{1}^{2} \mathrm{~d} \tau+4 \int_{0}^{\infty} \varrho(\tau) \mu(\tau)\langle u, \xi(\tau)\rangle_{1} \mathrm{~d} \tau .
$$

An integration by parts provides the equality (see [6, 16])

$$
2 \int_{0}^{\infty} \varrho(\tau) \frac{\mathrm{d}}{\mathrm{d} \tau}\|\xi(\tau)\|_{1}^{2} \mathrm{~d} \tau=-2\|\xi\|_{\mathcal{S}}^{2}
$$

Moreover, again using (17.2) and applying the Hölder and the Young inequalities,

$$
4 \int_{0}^{\infty} \varrho(\tau) \mu(\tau)\langle u, \xi(\tau)\rangle_{1} \mathrm{~d} \tau \leq \frac{4 M}{\delta}\|u\|_{1} \int_{0}^{\infty}\|\xi(\tau)\|_{1} \mathrm{~d} \tau \leq k\|u\|_{1}^{2}+\|\xi\|_{\mathcal{S}}^{2}
$$

where we set $k=\frac{4 M^{2} \kappa(0)}{\delta^{2}}$. Summarizing,

$$
\frac{\mathrm{d}}{\mathrm{d} t} \Psi+\|\xi\|_{\mathcal{S}}^{2} \leq k\|u\|_{1}^{2} .
$$

Finally, for $\varepsilon>0$ we define the energy functional

$$
\Phi(t)=E(t)+2 \varepsilon \Psi(t) .
$$

Up to fixing $\varepsilon$ suitably small, we get from (7.3) the uniform bounds

$$
E(t) \leq \Phi(t) \leq 2 E(t),
$$

whereas by collecting (7.1)-(7.4) we are led to the differential inequality

$$
\frac{\mathrm{d}}{\mathrm{d} t} \Phi+\varepsilon \Phi \leq b
$$

Thus, the Gronwall lemma allows us to conclude that

$$
E(t) \leq \Phi(t) \leq \Phi(0) \mathrm{e}^{-\varepsilon t}+\frac{b}{\varepsilon} \leq 2 E(0) \mathrm{e}^{-\varepsilon t}+\frac{b}{\varepsilon},
$$

and the claim follows by choosing $c=\frac{b}{\varepsilon}$.

A subsequent integration of inequality (7.1) on the time-interval $(t, t+1)$ provides a noteworthy corollary. 
Corollary 7.3. There exists an increasing positive function $\mathfrak{I}$ such that, for every $z \in \mathbb{B}_{r}$ and every $t \geq 0$,

$$
\|S(t) z\|_{\mathcal{H}}^{2}+\int_{t}^{t+1}\|u(y)\|_{1}^{2} \mathrm{~d} y \leq \Im(r) .
$$

\section{Exponential AND GLOBAL attractors}

Our main result, whose proof is given in the final Section 9 , concerns the existence of an exponential attractor for the semigroup $S(t)$ acting on $\mathcal{H}$ (see [19] for a detailed presentation of the subject). Roughly speaking, this is a small -in a suitable senseset which attracts exponentially fast all bounded subsets of $\mathcal{H}$ with respect to the Hausdorff semidistance. Recall that the Hausdorff semidistance between two (nonempty) sets $\mathcal{X}, \mathcal{Y} \subset \mathcal{H}$ is defined as

$$
\operatorname{dist}_{\mathcal{H}}(\mathcal{X}, \mathcal{Y})=\sup _{x \in \mathcal{X}} \inf _{y \in \mathcal{Y}}\|x-y\|_{\mathcal{H}}
$$

Theorem 8.1. There exists a set $\mathfrak{E} \subset \mathcal{H}$, called exponential attractor, satisfying the following properties:

(i) $\mathfrak{E}$ is compact with finite fractal dimension in $\mathcal{V}$

$$
\operatorname{dim}_{\mathcal{V}}(\mathfrak{E})=\limsup _{\varepsilon \downarrow 0} \frac{\ln N_{\varepsilon}(\mathfrak{E})}{\ln \frac{1}{\varepsilon}}<\infty,
$$

where $N_{\varepsilon}(\mathfrak{E})$ is the smallest number of $\varepsilon$-balls of $\mathcal{V}$ necessary to cover $\mathfrak{E}$.

(ii) $\mathfrak{E}$ is positively invariant under the action of the semigroup:

$$
S(t) \mathfrak{E} \subset \mathfrak{E}, \quad \forall t \geq 0 .
$$

(iii) For every $r \geq 0$ and every $t \geq 0$ the exponential attraction formula

$$
\operatorname{dist}_{\mathcal{H}}\left(S(t) \mathbb{B}_{r}, \mathfrak{E}\right) \leq \mathfrak{I}(r) \mathrm{e}^{-\omega t}
$$

holds for some $\omega>0$ and some increasing positive function $\mathfrak{I}$. Both $\omega$ and $\mathfrak{I}$ can be explicitly calculated.

By standard arguments (cf. 1, 4, 14, 15, 19, 21]), the existence of an exponential attractor, or more generally the one of a compact attracting set, implies the existence of the global attractor: the unique compact set $\mathfrak{A} \subset \mathcal{H}$ which is at the same time

- fully invariant: $S(t) \mathfrak{A}=\mathfrak{A}$ for every $t \geq 0$; and

- attracting: $\lim _{t \rightarrow \infty} \operatorname{dist}_{\mathcal{H}}\left(S(t) \mathbb{B}_{r}, \mathfrak{A}\right)=0$ for every $t \geq 0$.

In particular, since it is fully invariant, the global attractor $\mathfrak{A}$ is contained in every closed attracting set, such as $\mathfrak{E}$. This proves the next corollary.

Corollary 8.2. The semigroup $S(t)$ possesses the global attractor $\mathfrak{A}$. Moreover, $\mathfrak{A}$ is compact with finite fractal dimension in $\mathcal{V}$.

We also recall that, for an arbitrarily fixed $x \in \mathbb{R}$, the global attractor can be given the form (see [15])

$$
\mathfrak{A}=\{Z(x): Z \mathrm{CBT}\},
$$

where a complete bounded trajectory (CBT) of the semigroup is a function $Z \in$ $\mathcal{C}_{\mathrm{b}}(\mathbb{R}, \mathcal{H})$ satisfying

$$
Z(x)=S(t) Z(x-t), \quad \forall t \geq 0, \forall x \in \mathbb{R} .
$$


Quite interestingly, the trajectories lying on the attractor turn out to verify the formal equality (4.1) for all times.

Proposition 8.3. For every $\mathrm{CBT} Z=(u, \xi)$ we have the equality

$$
\xi^{x}(\tau)=\int_{0}^{\infty} \mu(\tau+s) u(x-s) \mathrm{d} s, \quad \forall x \in \mathbb{R} .
$$

Proof. If $Z=(u, \xi)$ is a CBT we know by definition that

$$
Z(x)=S(t)\left(u(x-t), \xi^{x-t}\right), \quad \forall t \geq 0, \forall x \in \mathbb{R} .
$$

Moreover, since $Z(x) \in \mathfrak{A}$ for all $x$,

$$
\sup _{x \in \mathbb{R}}\left[\|u(x)\|_{1}+\left\|\xi^{x}\right\|_{\mathcal{S}}\right]=K<\infty,
$$

for some $K>0$ depending only on $\mathfrak{A}$. Exploiting the representation formula (4.3), we get

$$
\xi^{x}(\tau)=\xi^{x-t}(t+\tau)+\int_{0}^{t} \mu(\tau+s) u(x-s) \mathrm{d} s .
$$

Accordingly, for an arbitrary $x \in \mathbb{R}$, we obtain the identity

$$
\xi^{x}(\tau)-\int_{0}^{\infty} \mu(\tau+s) u(x-s) \mathrm{d} s=\mathcal{Q}_{1}^{t}(\tau)+\mathcal{Q}_{2}^{t}(\tau),
$$

where

$$
\mathcal{Q}_{1}^{t}(\tau)=\xi^{x-t}(t+\tau) \quad \text { and } \quad \mathcal{Q}_{2}^{t}(\tau)=-\int_{t}^{\infty} \mu(\tau+s) u(x-s) \mathrm{d} s .
$$

Since the left-hand side above is independent of $t$, in order to conclude it is enough show to that $\mathcal{Q}_{\imath}^{t} \rightarrow 0$ in $\mathcal{S}$ as $t \rightarrow \infty$. Indeed, thanks to (2.3), we handle the first term as

$$
\left\|\mathcal{Q}_{1}^{t}\right\|_{\mathcal{S}}^{2}=\left\|L(t) \xi^{x-t}\right\|_{\mathcal{S}}^{2} \leq M \mathrm{e}^{-\delta t}\left\|\xi^{x-t}\right\|_{\mathcal{S}}^{2} \leq M K^{2} \mathrm{e}^{-\delta t} \rightarrow 0 .
$$

Concerning the latter one, we infer from (1.5) that

$$
\left\|\mathcal{Q}_{2}^{t}(\tau)\right\|_{1} \leq \int_{t}^{\infty} \mu(\tau+s)\|u(x-s)\|_{1} \mathrm{~d} s \leq M K \mu(\tau) \int_{t}^{\infty} \mathrm{e}^{-\delta s} \mathrm{~d} s=\frac{M K}{\delta} \mathrm{e}^{-\delta t} \mu(\tau) .
$$

In turn,

$$
\left\|\mathcal{Q}_{2}^{t}\right\|_{\mathcal{S}}^{2} \leq \frac{M^{2} K^{2}}{\delta^{2}} \mathrm{e}^{-2 \delta t} \int_{0}^{\infty} \nu(\tau)[\mu(\tau)]^{2} \mathrm{~d} \tau=\frac{M^{2} K^{2} \kappa(0)}{\delta^{2}} \mathrm{e}^{-2 \delta t} \rightarrow 0,
$$

as desired.

A quite direct consequence of Proposition 8.3 is the following result, whose proof is left to the reader.

Corollary 8.4. Given $u \in L^{\infty}\left(\mathbb{R}, \mathrm{H}^{1}\right)$ and defining $\xi=\xi^{x}(\tau)$ by formula (8.1), the function $Z=(u, \xi)$ is a CBT of $S(t)$ if and only if $u$ solves (in fact in the strong sense) the equation

$$
\dot{u}(x)+A\left[u(x)+\int_{0}^{\infty} \kappa(s) u(x-s) \mathrm{d} s\right]+\varphi(u(x))=f
$$

for every $x \in \mathbb{R}$. 


\section{Proof of Theorem 8.1}

The direct proof of the theorem requires several steps, some of which are rather technical. Nevertheless, an alternative and much more convenient strategy is possible, leaning on the earlier contributions in the history space framework. To this end, we first recall the main result of [2].

Theorem 9.1. There exists a set $\hat{\mathfrak{E}} \subset \hat{\mathcal{H}}$, compact with finite fractal dimension in $\hat{\mathcal{V}}$ and positively invariant for $\hat{S}(t)$, satisfying the exponential attraction formula

$$
\operatorname{dist}_{\hat{\mathcal{H}}}\left(\hat{S}(t) \hat{\mathbb{B}}_{r}, \hat{\mathfrak{E}}\right) \leq \hat{\mathfrak{I}}(r) \mathrm{e}^{-\hat{\omega} t}
$$

for some $\hat{\omega}>0$ and some increasing positive function $\hat{\mathfrak{I}}$, both explicitly calculated.

Remark 9.2. The growth restriction (1.2), not really necessary for the existence of the semigroup $\hat{S}(t)$, plays a crucial role here.

We now define the candidate exponential attractor of our problem as

$$
\mathfrak{E}=\Lambda \hat{\mathfrak{E}} \text {. }
$$

Due to the Lipschitz continuity of $\boldsymbol{\Lambda}$, we infer from Theorem 9.1 that $\mathfrak{E}$ is compact in $\mathcal{V}$ with fractal dimension

$$
\operatorname{dim}_{\mathcal{V}}(\mathfrak{E}) \leq \operatorname{dim}_{\hat{\mathcal{V}}}(\hat{\mathfrak{E}})<\infty
$$

Moreover,

$$
S(t) \mathfrak{E}=S(t) \boldsymbol{\Lambda} \hat{\mathfrak{E}}=\boldsymbol{\Lambda} \hat{S}(t) \hat{\mathfrak{E}} \subset \boldsymbol{\Lambda} \hat{\mathfrak{E}}=\mathfrak{E} .
$$

This establishes (i)-(ii). In order to prove the exponential attraction (iii), let $r \geq 0$ be fixed, and denote by $C_{r} \geq 0$ a generic constant depending (increasingly) only on $r$. Given a solution

$$
S(t) z=\left(u(t), \xi^{t}\right) \quad \text { with } \quad z=\left(u_{0}, \xi_{0}\right) \in \mathbb{B}_{r},
$$

we set (upon extending $u$ on $\mathbb{R}^{-}$equal to zero)

$$
\psi^{t}(s)=\int_{0}^{s} u(t-y) \mathrm{d} y
$$

Then we consider the function

$$
\hat{Z}(t)=\left(u(t), \psi^{t}\right)
$$

which satisfies the uniform-in-time estimate

$$
\|\hat{Z}(t)\|_{\hat{\mathcal{H}}} \leq C_{r} .
$$

Indeed, by Corollary [7.3, $\|u(t)\| \leq C_{r}$ and

$$
\left\|\psi^{t}(s)\right\|_{1}^{2} \leq\left(\int_{t-s}^{t}\|u(y)\|_{1} \mathrm{~d} y\right)^{2} \leq s \int_{t-s}^{t}\|u(y)\|_{1}^{2} \mathrm{~d} y \leq C_{r}\left(1+s^{2}\right) .
$$

Recalling (1.5), the latter inequality readily yields the remaining bound $\left\|\psi^{t}\right\|_{\mathcal{M}} \leq$ $C_{r}$. Finally, we introduce the function

$$
Z(t)=\Lambda \hat{Z}(t)=\left(u(t), \Lambda \psi^{t}\right) .
$$

Lemma 9.3. For every $x, y \geq 0$ we have

$$
\|S(x+y) z-S(x) Z(y)\|_{\mathcal{H}} \leq C_{r} \mathrm{e}^{\ell x-\frac{\delta}{2} y} .
$$


Proof. We first apply Proposition 4.3 to get

$$
\|S(x+y) z-S(x) Z(y)\|_{\mathcal{H}} \leq \mathrm{e}^{\ell x}\|S(y) z-Z(y)\|_{\mathcal{H}}=\mathrm{e}^{\ell x}\left\|\xi^{y}-\Lambda \psi^{y}\right\|_{\mathcal{S}} .
$$

Due to Lemma 2.3 (see also Remark 2.4),

$$
\left(\Lambda \psi^{y}\right)(\tau)=\int_{0}^{\infty} \mu(\tau+s) u(y-s) \mathrm{d} s=\int_{0}^{y} \mu(\tau+s) u(y-s) \mathrm{d} s .
$$

Thus, from (2.3) and the representation formula (4.3) for $\xi^{y}$, we find

$$
\left\|\xi^{y}-\Lambda \psi^{y}\right\|_{\mathcal{S}}^{2}=\left\|L(y) \xi_{0}\right\|_{\mathcal{S}}^{2} \leq M \mathrm{e}^{-\delta y}\|\xi\|_{\mathcal{S}}^{2} \leq C_{r} \mathrm{e}^{-\delta y},
$$

and the claim follows.

Lemma 9.4. For every $x, y \geq 0$ we have

$$
\operatorname{dist}_{\mathcal{H}}(S(x) Z(y), \mathfrak{E}) \leq C_{r} \mathrm{e}^{-\hat{\omega} x} .
$$

Proof. Exploiting Proposition 6.1 and Theorem 2.2, by the very definition of Hausdorff semidistance we obtain

$$
\operatorname{dist}_{\mathcal{H}}(S(x) Z(y), \mathfrak{E})=\operatorname{dist}_{\mathcal{H}}(\boldsymbol{\Lambda} \hat{S}(x) \hat{Z}(y), \boldsymbol{\Lambda} \hat{\mathfrak{E}}) \leq \operatorname{dist}_{\hat{\mathcal{H}}}(\hat{S}(x) \hat{Z}(y), \hat{\mathfrak{E}}) .
$$

As $\hat{Z}$ is uniformly bounded in $\hat{\mathcal{H}}$, the exponential attraction property of Theorem 9.1 completes the argument.

Conclusion of the Proof. Due to the straightforward inequality

$$
\operatorname{dist}_{\mathcal{H}}(S(x+y) z, \mathfrak{E}) \leq\|S(x+y) z-S(x) Z(y)\|_{\mathcal{H}}+\operatorname{dist}_{\mathcal{H}}(S(x) Z(y), \mathfrak{E}),
$$

setting for any $\varkappa \in[0,1]$

$$
x=\varkappa t \quad \text { and } \quad y=t-\varkappa t,
$$

we deduce from Lemma 9.3 and Lemma 9.4 that

$$
\operatorname{dist}_{\mathcal{H}}(S(t) z, \mathfrak{E}) \leq C_{r}\left[\mathrm{e}^{-\frac{1}{2}(\delta-2 \ell \varkappa-\delta \varkappa) t}+\mathrm{e}^{-\hat{\omega} \varkappa t}\right] .
$$

The optimal choice

entails

$$
\varkappa=\varkappa_{\star}=\frac{\delta}{2 \hat{\omega}+2 \ell+\delta}
$$

$$
\operatorname{dist}_{\mathcal{H}}(S(t) z, \mathfrak{E}) \leq C_{r} \mathrm{e}^{-\hat{\omega} \varkappa_{\star} t} .
$$

Since $z \in \mathbb{B}_{r}$ is arbitrary, the exponential attraction (iii) is attained with $\omega=$ $\hat{\omega} \varkappa_{\star}$.

\section{REFERENCES}

[1] A. V. Babin and M. I. Vishik, Attractors of evolution equations, Studies in Mathematics and its Applications, vol. 25, North-Holland Publishing Co., Amsterdam, 1992. Translated and revised from the 1989 Russian original by Babin. MR1156492 (93d:58090)

[2] Mickaël D. Chekroun, Francesco Di Plinio, Nathan E. Glatt-Holtz, and Vittorino Pata, Asymptotics of the Coleman-Gurtin model, Discrete Contin. Dyn. Syst. Ser. S 4 (2011), no. 2, 351-369, DOI 10.3934/dcdss.2011.4.351. MR2746378 (2012b:35164)

[3] V. V. Chepyzhov and V. Pata, Some remarks on stability of semigroups arising from linear viscoelasticity, Asymptot. Anal. 46 (2006), no. 3-4, 251-273. MR2215885 (2007c:47053)

[4] Vladimir V. Chepyzhov and Mark I. Vishik, Attractors for equations of mathematical physics, American Mathematical Society Colloquium Publications, vol. 49, American Mathematical Society, Providence, RI, 2002. MR1868930 (2003f:37001c)

[5] Bernard D. Coleman and Morton E. Gurtin, Equipresence and constitutive equations for rigid heat conductors, Z. Angew. Math. Phys. 18 (1967), 199-208 (English, with German summary). MR0214334 (35 \#5185) 
[6] Monica Conti and Elsa M. Marchini, Wave equations with memory: the minimal state approach, J. Math. Anal. Appl. 384 (2011), no. 2, 607-625, DOI 10.1016/j.jmaa.2011.06.009. MR2825211 (2012h:35224)

[7] Monica Conti, Elsa M. Marchini, and Vittorino Pata, Semilinear wave equations of viscoelasticity in the minimal state framework, Discrete Contin. Dyn. Syst. 27 (2010), no. 4, 1535-1552, DOI 10.3934/dcds.2010.27.1535. MR2629536(2011c:35059)

[8] Monica Conti, Vittorino Pata, and Marco Squassina, Singular limit of differential systems with memory, Indiana Univ. Math. J. 55 (2006), no. 1, 169-215, DOI 10.1512/iumj.2006.55.2661. MR2207550 (2006k:35290)

[9] Constantine M. Dafermos, Asymptotic stability in viscoelasticity, Arch. Rational Mech. Anal. 37 (1970), 297-308. MR0281400 (43 \#7117)

[10] Gianpietro Del Piero and Luca Deseri, On the concepts of state and free energy in linear viscoelasticity, Arch. Rational Mech. Anal. 138 (1997), no. 1, 1-35, DOI 10.1007/s002050050035. MR 1463802 (98i:73023)

[11] Luca Deseri, Mauro Fabrizio, and Murrough Golden, The concept of minimal state in viscoelasticity: new free energies and applications to PDEs, Arch. Ration. Mech. Anal. 181 (2006), no. 1, 43-96, DOI 10.1007/s00205-005-0406-1. MR2221203(2009a:74024)

[12] Claudio Giorgi, Vittorino Pata, and Alfredo Marzocchi, Uniform attractors for a nonautonomous semilinear heat equation with memory, Quart. Appl. Math. 58 (2000), no. 4, 661-683. MR1788423 (2001k:37129)

[13] H. Grabmüller, On linear theory of heat conduction in materials with memory. Existence and uniqueness theorems for the final value problem, Proc. Roy. Soc. Edinburgh Sect. A 76 (1976/77), no. 2, 119-137. MR0446112 (56 \#4444)

[14] Jack K. Hale, Asymptotic behavior of dissipative systems, Mathematical Surveys and Monographs, vol. 25, American Mathematical Society, Providence, RI, 1988. MR.941371 (89g:58059)

[15] Alain Haraux, Systèmes dynamiques dissipatifs et applications, Recherches en Mathématiques Appliquées [Research in Applied Mathematics], vol. 17, Masson, Paris, 1991 (French). MR:1084372 (92b:35002)

[16] Mauro Fabrizio, Claudio Giorgi, and Vittorino Pata, A new approach to equations with memory, Arch. Ration. Mech. Anal. 198 (2010), no. 1, 189-232, DOI 10.1007/s00205-010-0300-3. MR.2679371(2011e:35393)

[17] S.-O. Londen and J. A. Nohel, Nonlinear Volterra integro-differential equation occurring in heat flow, J. Integral Equations 6 (1984), no. 1, 11-50. MR727934 (85j:45027)

[18] R. K. Miller, An integro-differential equation for rigid heat conductors with memory, J. Math. Anal. Appl. 66 (1978), no. 2, 313-332, DOI 10.1016/0022-247X(78)90234-2. MR515894 (80g:45015)

[19] A. Miranville and S. Zelik, Attractors for dissipative partial differential equations in bounded and unbounded domains, Handbook of differential equations: evolutionary equations. Vol. IV, Handb. Differ. Equ., Elsevier/North-Holland, Amsterdam, 2008, pp. 103-200, DOI 10.1016/S1874-5717(08)00003-0. MR2508165 (2010c:37175)

[20] Jace W. Nunziato, On heat conduction in materials with memory, Quart. Appl. Math. 29 (1971), 187-204. MR0295683 (45 \#4749)

[21] Roger Temam, Infinite-dimensional dynamical systems in mechanics and physics, Applied Mathematical Sciences, vol. 68, Springer-Verlag, New York, 1988. MR953967 (89m:58056)

Dipartimento di Matematica "F.Brioschi", Politecnico di Milano, Via Bonardi 9, 20133 Milano, Italy

E-mail address: monica.conti@polimi.it

Dipartimento di Matematica "F.Brioschi", Politecnico di Milano, Via Bonardi 9, 20133 Milano, Italy

E-mail address: elsa.marchini@polimi.it

Dipartimento di Matematica "F.Brioschi", Politecnico di Milano, Via Bonardi 9, 20133 Milano, ItAly

E-mail address: vittorino.pata@polimi.it 